\title{
Toward a dynamic analysis of bipedal robots inspired by human leg muscles
}

\author{
B. Fernini ${ }^{1,2}$, M. Temmar ${ }^{2}$ and M.M. Noor ${ }^{3}$ \\ ${ }^{1}$ Centre universitaire de Tissemsilt. Algeria. \\ ${ }^{2}$ Laboratory of structures, Department of Mechanical Engineering, University of Blida1, \\ Algeria \\ ${ }^{3}$ Faculty of Mechanical Engineering, Universiti Malaysia Pahang (UMP), Malaysia, \\ ${ }^{1}$ Email: fernini.brahim12@gmail.com \\ Phone : (+213)550962793
}

\begin{abstract}
A walking bipedal robot by adding passive springs like mono and biarticular muscles which correspond to rectus femoris (RF), biceps femoris (BF), gastrocnemius (GAS) and tibialis anterior (TA) in human legs has been modeled in this paper. The stability of human-like leg walking can be achieved by adding these passive springs in the leg mechanism of bipedal robot. On the other hand, using these springs may inflict a fatigue during walking due to the additional work that can provide to the joints. The main objective of this paper is to analyze the total work of the robot during walking by proposing four cases of the preload of the springs at the equilibrium position. It's found from this study that the case that has the most energy saving and ensure the comfortable walking to the robot is when the two muscles (GAS) and (TA) are not preloaded to support the total weight at the equilibrium position.
\end{abstract}

Keywords: Walking bipedal robot; mono and biarticular muscles; total work; newton euler; zero moment point; energy saving; comfortable walking; walking fatigue.

\section{INTRODUCTION}

Human walking has been extensively studied in the field of biomechanics [1]. For example, in the fields of biomechanics and robotics, bipedal walking has been investigated for our further understanding of adaptive locomotion mechanisms of human and robots [2]. Obtaining human-like robotic walking has been a long standing, if not always explicitly stated goal of robotic locomotion. The quality of metal machining also important to ensure the quality of the parts [3]. Achieving human-like robotic walking goal promises to result in robots able to navigate the myriad of terrains that humans can handle with ease; this would have, for example, important applications to space exploration [4]. Moreover, going beyond purely robotic systems, if one can understand how to make robots walk-like humans, this understanding can be used to build robotic assistive and prosthetic devices to aid people with walking impairments and lower extremity amputations walk more efficiently and naturally [4-7] thus, the ability to obtain human-like robotic walking has important and far-reaching ramifications [4]. From the 
biomimetic point of view, it is valuable to look at the walking behaviors of humans in order to achieve a comfortable robotic walking $[8,9]$.

Research on biologically inspired robots is advancing nowadays. Many industrial robots, which are inspired by human leg muscles, are used for performing many tasks [2, 10-13]. Such muscles are categorized in two types: mono and biarticular muscles, which have the following properties [12, 14]:

i. Monoarticular muscles: these muscles are connected to two links on each side of single joint. They can drive only one joint by producing torque around that joint.

ii. Biarticular muscles: these muscles are connected to two links separated by a third link and two joints and can drive these two joints simultaneously, and have the capability to transfer energy mainly produced by monarticular muscles to joints where they can effectively contribute to the desired aim of movement.

The mono and biarticular muscles that correspond to rectus femoris (RF), biceps femoris (BF) gastrocnemius (GAS) and tibialis anterior (TA) in human legs play significant roles in the stability of human walking $[10,11]$. The muscles (RF) is a bifunctional muscle responsible for hip flexion in the swing phase and knee extension in the late swing and stance phase, the (BF) muscle responds to the hip extension in the stance phase and the knee flexion in the swing phase the (GAS) muscle is primarily responsible for ankle plantarflexion but also takes a minor role in knee extension, the TA muscle has two distinct roles during human walking: (1) to dorsiflex the ankle during the swing phase for foot clearance and placement; (2) to contract during ankle plantarflexion at the initial foot contact with the ground $[1,15]$. Thus, using springs like mono-biarticular muscles in the leg mechanism of bipedal robots are able to achieve the stability of human-like leg walking compared with those that assume the bipedal robots like rigid body structures.

On the other hand, using these springs may inflict a serious damage of the leg mechanism and causes the walking fatigue of the robot due the additional work that can provide to the joint legs during the walk period of bipedal robots [16]. In this sense, we need to check the total work of joints of each complaint leg during walking. In addition, the works analysis at joints are necessary to deal with the issue of comfortable walking for bipedal robotic applications [9]. However, it actually requires significant effort to evaluate the comfortability of walking which is closely related to obtaining good performance of legged robots [9, 16-19]. In biped robots, springs may be used to support the weight of the robot itself or its weight and a supplementary charge, or to manage energy (accumulating energy during landing phase and reinjecting it during a lifting phase). In this paper, the focus is on the weight support.

The objective of this paper is to model the walking bipedal robot by adding springs like mono and biarticular passive muscles and to study the effect of those muscles on the total work of each leg during the walk. For this purpose, our paper is organized as follows: in section 2, we model the walking bipedal robot with springs like mono and biarticular muscles. In section 3: is devoted to the discussion of obtained simulation results to show the influence of the muscles on the total work of each leg, and the conclusion is drawn in the section 4 .

\section{MODELING OF A BIPEDAL ROBOT WITH COMPLIANT LEG}

In order to have an effective analysis on the effect of mono and biarticular muscles on the total work of each leg of robot during walking, we consider the bipedal robot model with springs like mono and biarticular muscles as shown in Figure 1. The bipedal model shown 
in Figure 1 consists of four leg limbs $\left(H_{1} H_{2}, H K, A K_{1}\right.$ and $\left.B C\right)$ with upper body, three joints (hip, knee and ankle joints) and four linear springs, which are represented by the red dashed lines. The springs S1, S2, S4 correspond to the biarticular muscles: rectus femoris (RF), biceps femoris (BF) and gastrocnemius (GAS) in human legs respectively. Additionally, the spring S3 that correspond to a monoarticular muscle: tibialis anterior (TA) in human legs is also used in this model. The force generated in these springs $f=\left[f_{1}, f_{2}, f_{3}, f_{4}\right]$ is calculated by the scalar product of the stiffness coefficient and the displacement (lengthening or compression) of spring, and in our case the intrinsic damping factor of springs is neglected. The point $P=\left[H_{1}, H_{2}, K_{1}, E, F, G, C, B\right]$ represent the spring attachment. The center of mass of the system is located at the hip joint $(H)$. The limb mass is defined at the center of each limb. The two limbs $\left(H_{1} H_{2}\right)$ and $(B C)$ are considered in our model to remain parallel during the motion. The $\left(H_{1} H_{2}\right)$ represents the upper body attached to the hip joint. The $(B C)$ refers to the foot in contact with the horizontal ground or moving parallel to it.

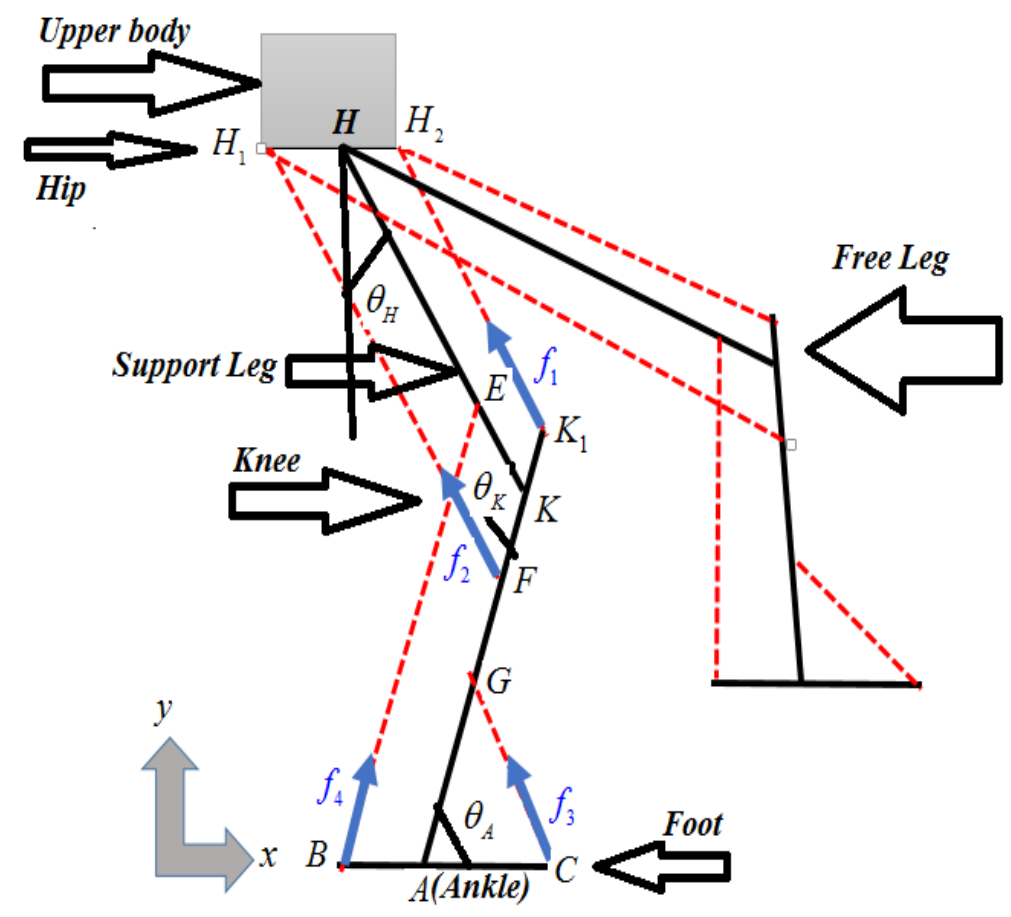

Figure 1. Bipedal locomotion model with compliant legs.

The dynamic simulations are realized with Matlab/Simulink by using Newton-Euler method [20] and the joint work is calculated by the integral of the scalar product of the joint torque and the joint angle [21]. Simulations have been performed separately for each leg, with a 2D model, to allow easy analysis of the results by decoupling the behaviour of each subsystem instead of analyzing only the global system. The motion simulated by using ZMP method [22-23] correspond to walking bipedal robot in straight line at a constant velocity of $2 \mathrm{~m} / \mathrm{s}$ with the hip at a constant height of $0,49 \mathrm{~m}$ (no vertical acceleration). The support foot does not slip on the contact surface (coefficient of friction $=0.5$ ) . For the simulation by using AMEsim and Matlab/Simulink, the dimensions and mass of segments of the robot are shown in [22]. 


\section{RESULTS AND DISCUSSION}

This section discusses the outcomes of the work and the results were drawn in Figure 2.This Figure shows the simulation of walking bipedal robot by using AMEsim for a step $(0.36 \mathrm{~s})$. The short double support phase has been excluded. The right leg is the support leg during the motion, and the left leg is the free leg. The nominal inputs correspond to the offline trajectory computed with Matlab, while the realized outputs correspond to the results of a dynamical simulation performed with AMESim. In AMESim, PIDs are used to guarantee a minimum joint error and the nominal and realized-curves are almost coincident.

\section{Right Leg}

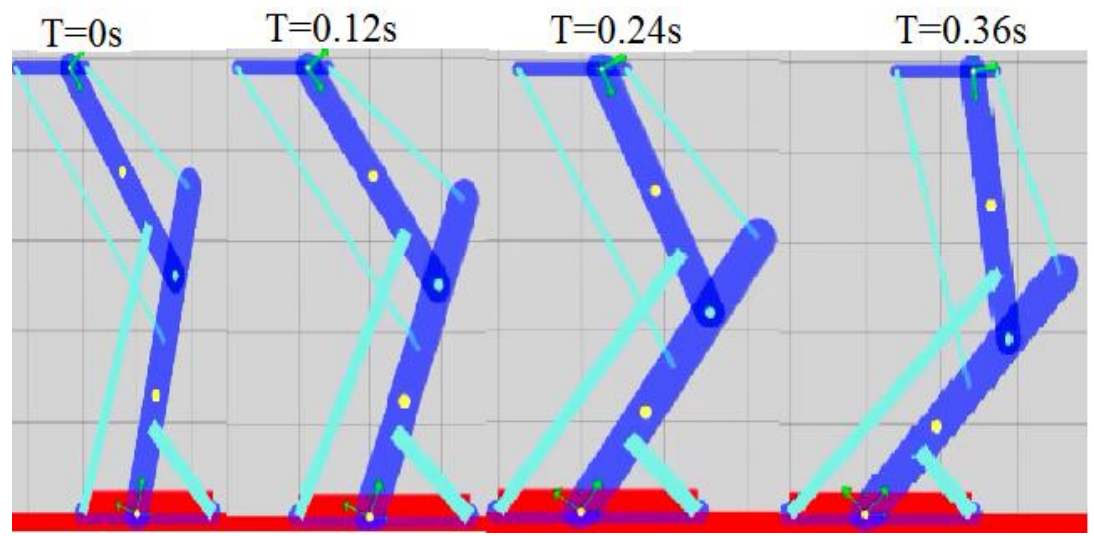

\section{Left Leg}

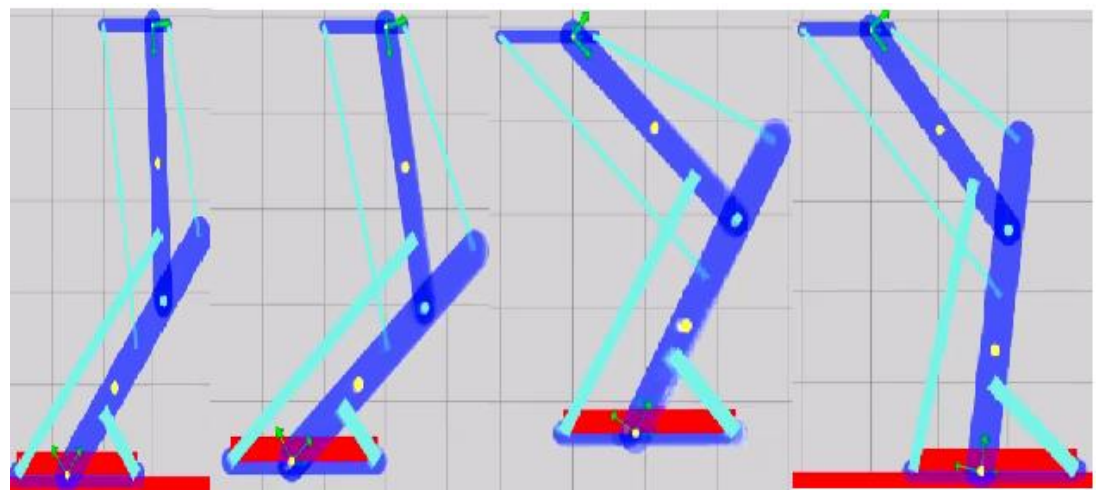

Figure 2. Motion simulation of walking bipedal robot by using AMEsim.

For simplicity and ease of comparison, the four springs use the same stiffness $\left(k_{1}=k_{2}=\right.$ $k_{3}=k_{4}=k$ ), but four different values of $k$ have been used, as explained below. The equilibrium position of bipedal robot has been defined with the hip at the vertical of the ankle (same $(x)$ coordinate for the hip and ankle) [22-23].

In order to analyze the effect of springs on the work the motors have to supply, four cases have been simulated:

Case (1): Arbitrary preload on the all muscles at the equilibrium position $\left(\mathrm{F}_{\mathrm{RF}}=-1000 \mathrm{~N}\right.$, $\mathrm{F}_{\mathrm{BF}}=-291 \mathrm{~N}, \mathrm{~F}_{\mathrm{GAS}}=-1156.5 \mathrm{~N}$ and $\mathrm{F}_{\mathrm{TA}}=-1000 \mathrm{~N}$ ). 
Case (2): the same thing like case (1), but in this case the preload of (TA) and (GAS) are used to support the total weight of the robot at the equilibrium position. ( $F_{\mathrm{RF}}=-1000 \mathrm{~N}$, $\mathrm{F}_{\mathrm{BF}}=-291 \mathrm{~N}, \mathrm{~F}_{\mathrm{GAS}}=-2015 \mathrm{~N}$ and $\left.\mathrm{F}_{\mathrm{TA}}=-1675 \mathrm{~N}\right)$.

Case (3): in this case: at the equilibrium position, the preload on the two muscles (RF) and (BF) are used to support the half of the rest of the body and the preload on the two muscles (GAS) and (TA) are used to support the total weight of the robot. $\left(F_{\mathrm{RF}}=-265 \mathrm{~N}\right.$, $\mathrm{F}_{\mathrm{BF}}=-77 \mathrm{~N}, \mathrm{~F}_{\mathrm{GAS}}=-1394 \mathrm{~N}$ and $\left.\mathrm{F}_{\mathrm{TA}}=-1159 \mathrm{~N}\right)$.

Case (4): in this case: at the equilibrium position, the preload on the two muscles (RF) and $(\mathrm{BF})$ are used to support the entire of the rest of the body weight and the preload on the two muscles (GAS) and (TA) are used to support the total body weight $(B W)$. $\left(\mathrm{F}_{\mathrm{RF}}=-\right.$ $530 \mathrm{~N}, \mathrm{~F}_{\mathrm{BF}}=-154 \mathrm{~N}, \mathrm{~F}_{\mathrm{GAS}}=-1618 \mathrm{~N}$ and $\left.\mathrm{F}_{\mathrm{TA}}=-1345 \mathrm{~N}\right)$.

The preload is realized by pre-compression of the springs in the equilibrium position. This means that in the equilibrium position, with the nominal stiffness chosen, the system is in static equilibrium. The nominal stiffness has been arbitrary set to $5000 \mathrm{~N} / \mathrm{m}$. It has also been decided that the equilibrium would be realized by the leg support alone. This means that the total supported weight at the hip is the upper body's weight and the weight of the free leg. The three other values of stiffness used in simulations are : $0 \mathrm{~N} / \mathrm{m}$, this corresponds to no springs at all, like the original bipedal robot; $2500 \mathrm{~N} / \mathrm{m}$, this is half the nominal stiffness and this also corresponds approximately to the equilibrium with the two feet on the ground sharing the weight of the upper body; $10000 \mathrm{~N} / \mathrm{m}$, this is twice the nominal stiffness and it allows to analyse what happens if one thinks of a very rigid system allowing to bear a charge without using the motor drives. This three stiffness are used with the same pre-compression (same deformation of springs at the equilibrium position) as the one use with the nominal stiffness.

We will now look at some results which are not shown in this paper. First, the spring forces during the motion (only the spring forces of case (1) and case (2) is shown in this paper). The spring forces depend only on the configuration (joint angles) of the system during the motion. For the support leg, (BF) and (RF) behave the same way for case (1) and (2) because the pre-constrained is the same for the two cases. The mass center of the upper body is directly placed at the hip joint, in such a way that the hip motor should not have to work to maintain the upper body $\left(\mathrm{H}_{1} \mathrm{H}_{2}\right)$ parallel to the ground if the robot were accelerating. The (RF) and (BF) forces in the neutral position do not generate a net torque in $(H)$. They generate a reaction (torque) that is supposed to prevent the rotation of the body. The pre-constraints also have the effect of lifting the body (they push on the body $\left(H_{1} H_{2}\right)$ and the pivot $(H)$ must pull down). All the curves cross the $(x)$ axis around $0.17 \mathrm{~s}$ (more precisely $0.1708 \mathrm{~s}$ ), and this correspond to the passage at the equilibrium position. At the start of the motion ( $H$ behind $A$ for the support leg) and all muscles are almost always compressed. The behaviour for the free leg is similar, with higher limit (shape curves due to the fact that the foot rises about $78 \mathrm{~mm}$ above de ground, giving rise to higher elongation or compression of the springs. These results don't give information on how these forces act to equilibrate or not the system (analysis is not as direct as with mono-articular muscles only), however it is clear that a higher spring stiffness gives rise to higher forces.

The second point of focus is the joint driving torques required to produce the motion. The joint torque patterns of the right leg for the two cases show that the hip torque pattern is the same for the two cases and is proportional to the stiffness coefficient. Also without springs $(k=0 \mathrm{~N} / \mathrm{m})$ there should be no torque required. In case (1), spring stiffness has no effect on the knee joint torque. Initial oscillations have been appeared due to footground compliance. For case (2), the nominal preload (with $k=5000 \mathrm{~N} / \mathrm{m}$ ) brings the knee torque around 0 N.m compared to an average around 70 N.m without springs. This is the weight support effect looked for. The ankle torque patterns for the two cases show that the magnitudes of the ankle torque for case (2) are higher than for case (1). Thus, we can conclude through the joint torque analysis that the preload of (GAS) and (TA) has 
effects only on the behavior of the knee and ankle torques. This is also true for the free leg, although the patterns are quite different.

Finally, we analyze the work done by each individual motor drive and the resulting total work. Figure 3 and Figure 4 show the total work (sum of the work of the three motors) for the support leg for case (1) and case (2). In case (1), the effect of springs is quite negligible with a little increase of the total work due mainly to the hip joint. In case (2), Figure4 shows a significant reduction of the total work with the nominal spring (remember that the system was balanced with $k=5000 \mathrm{~N} / \mathrm{m}$ ). This reduction due to the value of the joint torque of the knee which is around $0 \mathrm{~N} / \mathrm{m}$ as shown above.

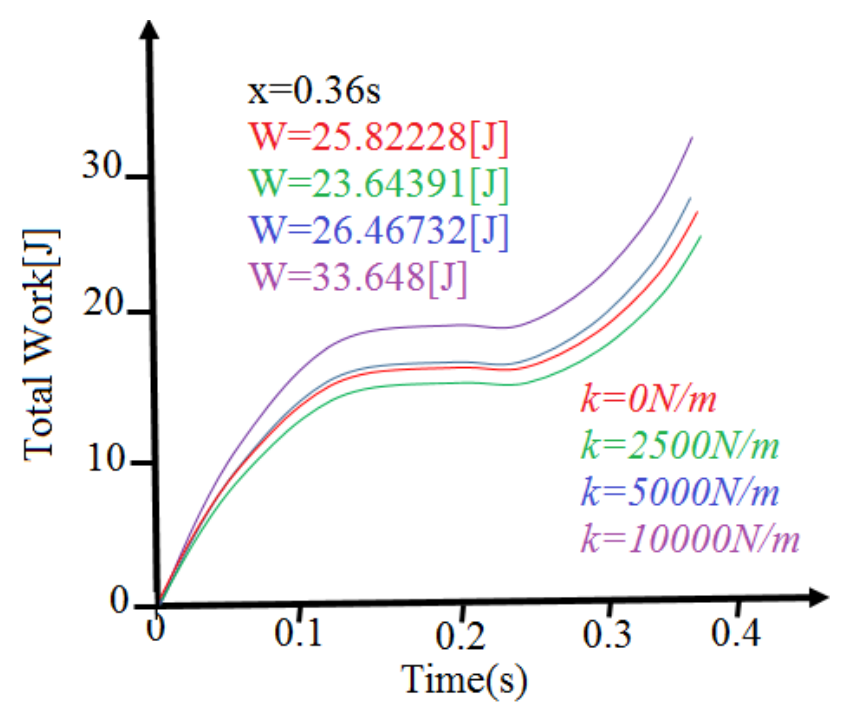

Figure 3. The total works of the right leg case (1).

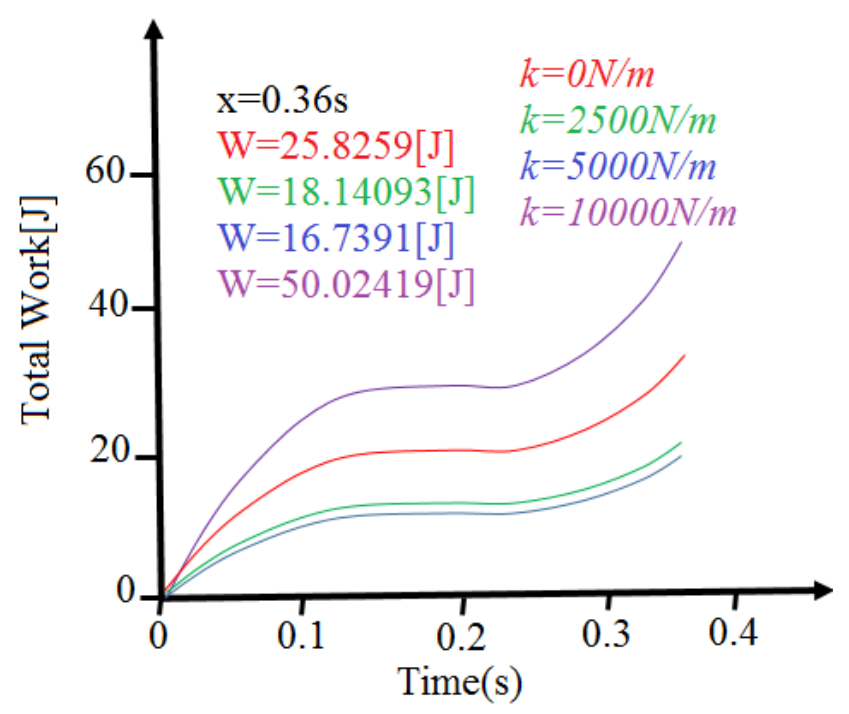

Figure 4. The total works of the right leg case (2).

The total work required by the support leg is reduced by $63.24 \%$. However, taking into account the work required by the free leg (left leg, Figures 5 and 6 for cases (1) and (2)), one can see that in either cases, there is a significant increase of the total 
work to move the free leg. Always by considering the nominal stiffness, this increase reaches a dramatic $202.27 \%$ for the free leg when (GAS) and (TA) are preloaded to support the entire robot system at the equilibrium position and combining the work of the two legs results in a net increase of $139.03 \%$. If one considers the case where the equilibrium is realized with the two legs on the ground in case (2) $(\mathrm{k}=5000 \mathrm{~N} / \mathrm{m})$, the total networks would be about $104.63 \%$. The total net works for the two cases in the case where $(\mathrm{k}=10000 \mathrm{~N} / \mathrm{m})$, show is not interesting to oversize springs, it provides no benefits.

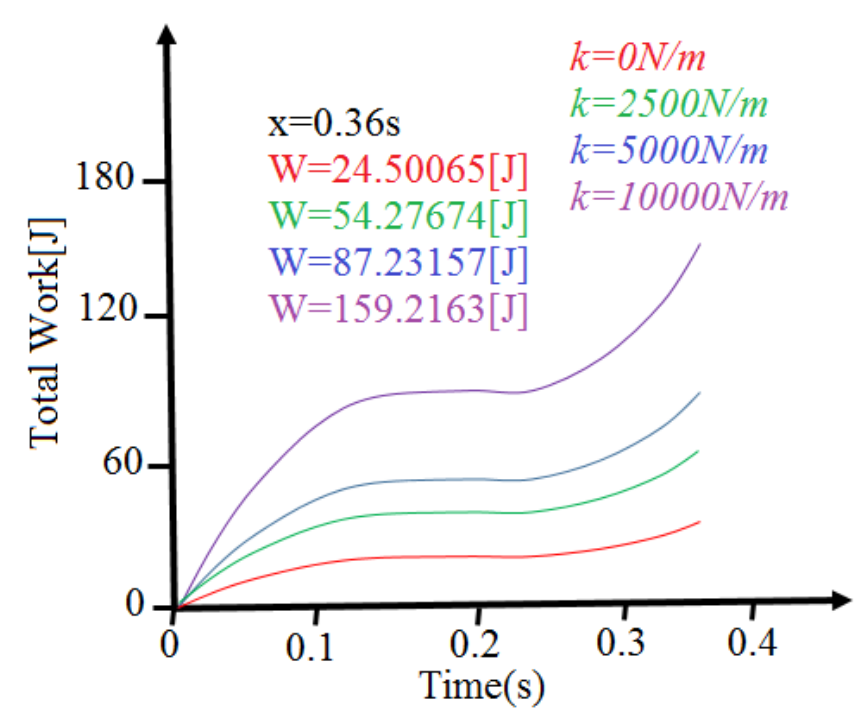

Figure 5. The total works of the left leg case (1).

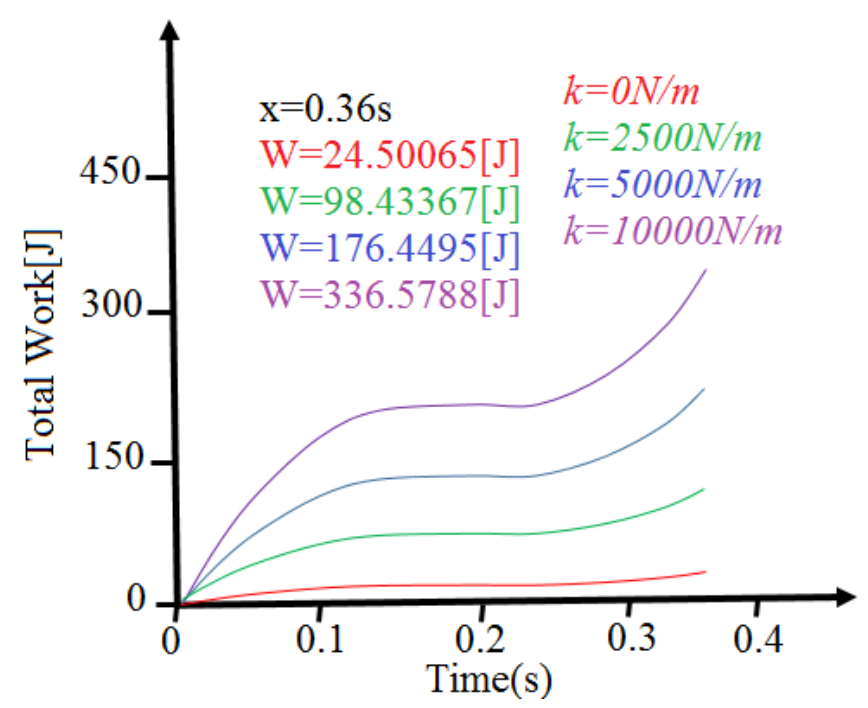

Figure 6. The total works of the left leg case (2).

Another fact that can be drawn from this paper is that the use of the preload on the two springs (RF) and (BF) to support the rest of the body at the equilibrium position decreases the total net work of the robot by $3.42 \%$ compared with the use of the preload on the two springs (RF) and (BF) to support the half of the rest of the body 
weight at the equilibrium position. This important result is clear from the Figures 7, 8, 9 and 10. As known, the factor "energy saving" plays an important role in robotic field [24-26]. If we compare between the four cases from the point of view "energy saving", it's very clear that in the case (1) the energy provided by the robot to move the leg mechanism is less than the other cases with around $30 \%$ of energy reduction. This means that the use of case (1) guarantees a comfortable walking and a good performance to the robot in comparison with the other cases. In this paper we have only studied the total work in one-step of walking $(\mathrm{T}=0.36 \mathrm{~s})$ and the focus on energy saving because the total work increases gradually during the walk period of bipedal robot as shown in [16].

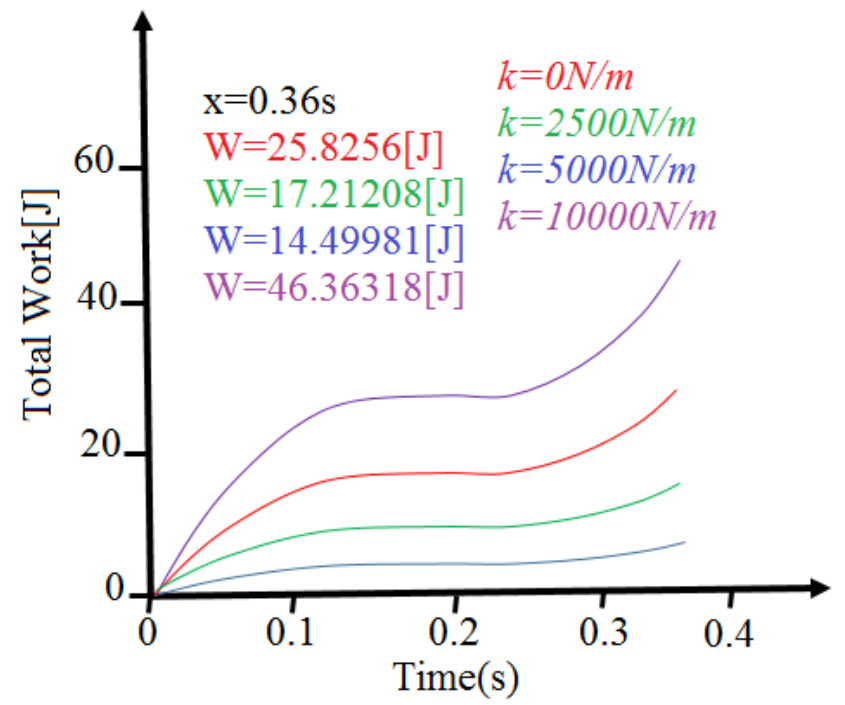

Figure 7. The total works of the right leg case (3).

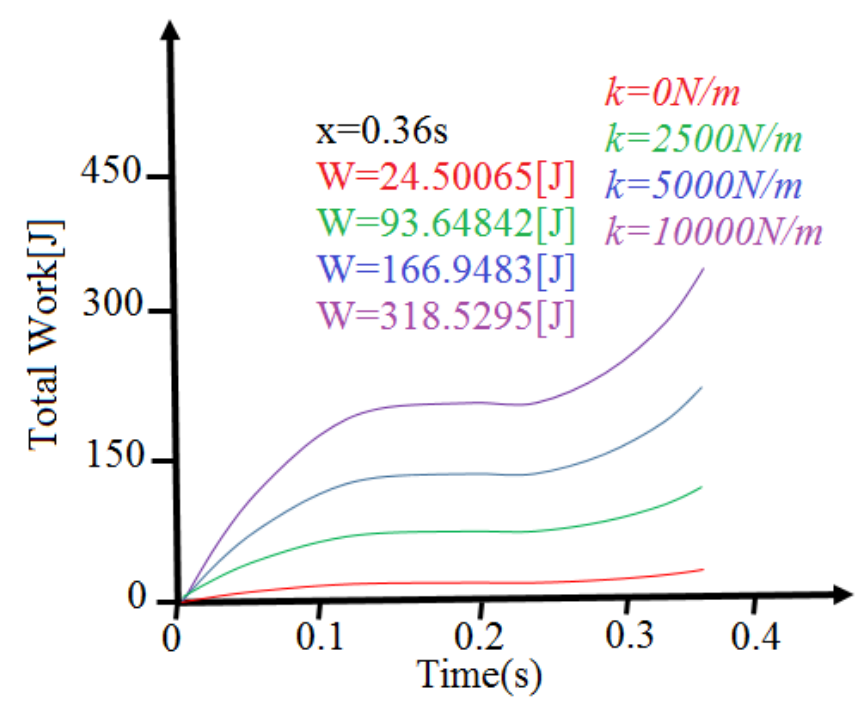

Figure 8. The total works of the left leg case (3). 


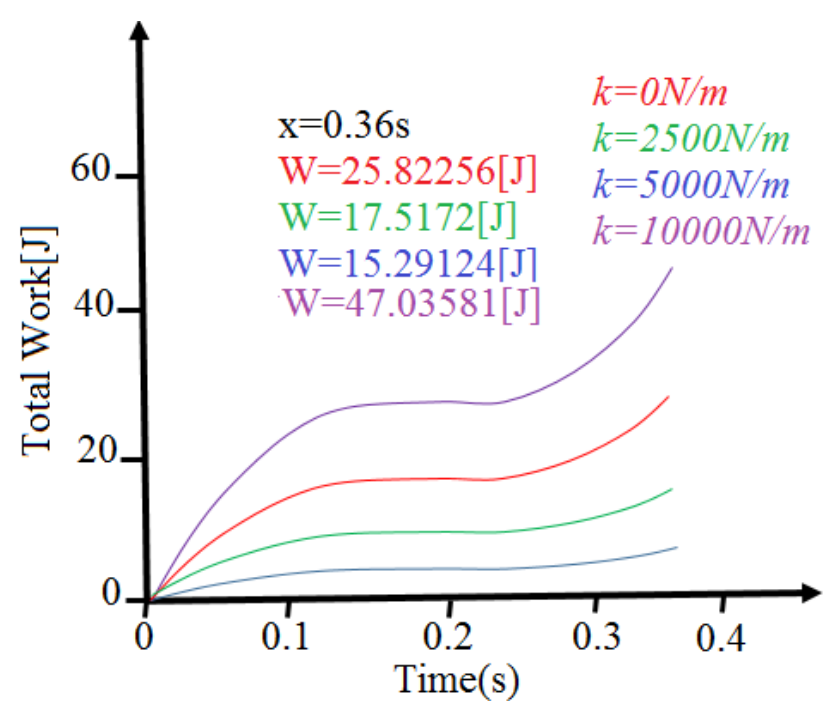

Figure 9. The total works of the right leg case (4).

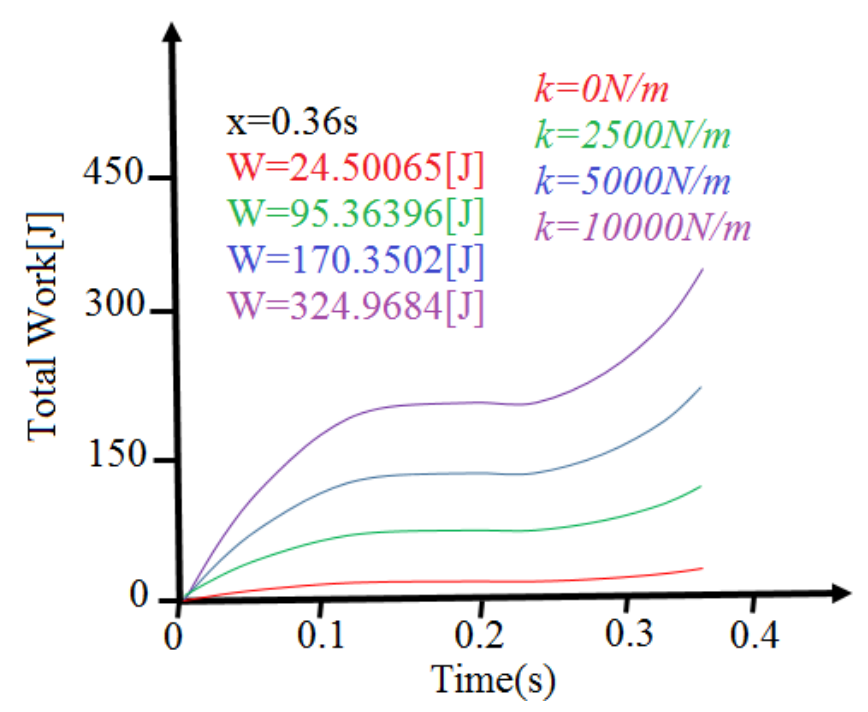

Figure 10. The total works of the left leg case (4).

\section{CONCLUSION AND PERSPECTIVE}

This paper addressed one of the scientific gaps of the dynamic walking locomotion via the dynamic analysis of the walking bipedal robot with mono and biarticular passive muscles. A comparison of the total work of the compliant legs found in this study with those of other studies was not possible, since these results have not been reported by other studies on the effect of mono and biarticular muscles on the joint works of bipedal robots. The novelty and perspective of this analysis can be drawn as follow:

- Using mono and biarticular passive springs in the leg mechanism of bipedal robot has a direct effect on the comfortability of the robot during walking and the choice of a good configuration of these springs from the point of view " energy saving" guarantees to the robot a comfortable walking.

- The use of spring-like mono and biarticular muscles to self-support the weight of the robot may be interesting if the robot has to stand for long periods because 
almost no energy would be spent by the motors but if the robot would have to walk more than $45 \%$ of the time, the use of springs is not recommended, based on the energy criterion.

- Other configurations and walking pattern may also lead to different conclusions and one must be careful in extrapolating the results presented here. It could be interesting also if a mechanism could be used for the free leg to engage/disengage springs in a way to manage energy while the leg is not in support phase instead of creating resistance to motion. This opens the door to future works.

\section{ACKNOWLEDGEMENTS}

This work has been done at Takanishi Laboratory-Waseda University-Tokyo-Japan, and the authors would like to thank Dr. Aiman Omar from Takanishi lab for his help and assistance, and Pr. Benoit Levesque from Laval University for the simulation performed with AMESIM. Thanks also to Centre Universitaire de Tissemsilt, University of Blida 1 and Universiti Malaysia Pahang (www.ump.edu.my) for the laboratory and financial support.

\section{REFERENCES}

[1] M Lin, A Catherine, P Bernd, G Henrik. Bipedal robotic walking control derived from analysis of human locomotion. Springer Biological Cybernetics. 2018.

[2] F Lida, Y Minekawa, J Rummel, A Seyfarth. Toward a human-like biped robot with compliant legs. Robotics and Autonomous Systems. 2009; 57(2): 139-144.

[3] K Kadirgama, MM Noor, Ahmed NA Alla, End milling surface roughness optimization using response ant colony, Sensors, 2010, 10, pp: 2054-2063

[4] D Aaron. First steps toward automatically generating bipedal robotic walking from human data. Chapter 8 Springer Robot Motion and Control Volume 422 of the Series Lecture Notes in Control and Information Sciences. 2011; 89-116.

[5] S Srinivasan, E Westervelt, A Hansen. A low-dimensional sagittal-plane forward-dynamic model for asymmetric gait and its application to study the gait of transtibial prosthesis users. ASME Journal of Biomechanical Eng. 2009; 131.

[6] P Dilworth, H Herr. An ankle foot emulation system for the study of human walking biomechanics. IEEE Intelligent Conference on Robotics and Automation. 2006; 2939-2945.

[7] P Sauer, K Kozlowski, Y Morita, H Ukai. Ankle robot for people with drop foot case study. Springer Robot Motion and Control, Lecture Notes in Control and Information Sciences. 2009; 396: 443-452.

[8] CC Norkin, PK Levangie. Joint structure \& function. Published by F.A Davis Company. 1992. 
[9] HK Byoung. Work analysis of compliant leg mechanisms for bipedal walking robots. International Journal of Advanced Robotic Systems. 2013; 10:334.

[10] F Lida, J Rummel, A Seyfarth. Bipedal walking and running with spring-like biarticular muscles. Journal of Biomechanics. 2008; 41(3): 656-667.

[11] RD Katayon. Advancing musculoskeletal robot design for dynamic and energy efficient bipedal locomotion. Vom Fachbereich Informatik der Technischen Universität Darmstadt zur Erlangung des akademischen Grades einer DoktorIngenieurin (Dr.-Ing.) genehmigte. 2014.

[12] D Emre. Walking motion control of biped robotic legs inspired by human muscle model for adapting variation of vertical load. Doctorat Thesis Tokyo University. 2012.

[13] AS Maziar, R Christian, K Stefan, S Dorian, M Rico, R Katayon, Z Guoping, MR Aida, V Oskar, S Andre. A new biarticular actuator design facilitates control of leg function in BioBiped3. IOP Science Bioinspiration \& Biomimetics. 2016.

[14] N Yoshihiro, I Atsuhiro, N Yutaka. Hopping of a monopedal robot with a biarticular muscle driven by electromagnetic linear actuators. IEEE ICRA. 2012.

[15] CA Macleod, L Meng, BA Conway, B Porr. Reflex control of robotic gait using human walking data. PloS ONE. 2014.

[16] B Fernini, M Temmar. The positions effect of biarticular muscles on the walking fatigue of bipedal robots. RDM Tissemsilt. 2016.

[17] DA Messuri, CA Klein. Automatic body regulation for maintaining stability of a legged vehicle during rough-terrain locomotion. IEEE Journal on Robotics and Automation. 1985; RA-1(3): 132-141.

[18] E Garcia, P Gonzalez. An improved energy stability margin for walking machines subjects to dynamic effects. Robotica. 2005; 23(1):13-20.

[19] T Bretl, S Lall. Testing static equilibrium for legged robots. IEEE Transactions on Robotics. 2008; 24(4): 794-807.

[20] BH Erin, OR Patrick, EK David. A dynamic analysis of the joint forces and torques during rising from a chair. IEEE Transactions on Rehabilitation Engineering. 1994.

[21] MZ Vladimir. Kinetics of Human Motion. The Pennsylvania University USA. 2002; ISBN: 0-7360-3778-0.

[22] O Aiman. Study of a biped robot locomotion on planets with different gravities. Doctorat Thesis Takanishi Laboratory-Waseda University Tokyo. 2012.

[23] B Fernini, M Temmar. The effect of mono and biarticular muscles on the dynamic of walking bipedal robot. IEEE Intelligent Systems Conference (IntelliSys). 2017; 969-978. 
[24] M Gouasmi, M Ouali, B Fernini, M Meghatria. Kinematic modelling and simulation of a 2-R robot using solidworks and verification by MATLAB/Simulink. International Journal of Advanced Robotic Systems. 2012.

[25] B Fernini, M Temmar. An algorithm for serial planar manipulators that have an architecture R//(n) R by using Solidworks (2013) and Matlab/Simulink (2012). IEEE International Conference on Mechatronics and Automation (ICMA). 2013.

[26] B Fernini, M Temmar. Dynamic Behavior of a SCARA Robot by using NE Method for a Straight Line and Simulation of Motion by using Solidworks and Verification by Matlab/Simulink. IAES International Journal of Robotics and Automation. 2014. 